\title{
The interface between general adult psychiatry and behavioural neurology/neuropsychiatry
}

\author{
Ketan Dipak Jethwa, Verghese Joseph, Vivek Khosla \& Andrea E. Cavanna
}

\begin{abstract}
SUMMARY
With the subspecialisation of psychiatry in the UK, clinicians may encounter problems at the interface between specialties. This article focuses on the interface between general adult psychiatry and behavioural neurology/ neuropsychiatry. Important interface issues include the identification of psychiatric symptoms in patients with organic brain pathology and the remit and organisation of general and specialist services. Options for optimum cooperation are presented alongside practical examples of clinical issues of neuropsychiatric relevance.
\end{abstract}

\section{LEARNING OBJECTIVES}

- Develop an understanding of the roles of the behavioural neurologist and neuropsychiatrist

- Develop an understanding of important issues at the interface between general adult psychiatry and behavioural neurology/neuropsychiatry

- Be aware of options for optimum cooperation at the interface

\section{DECLARATION OF INTEREST}

None

\section{Clinical vignette}

A 51-year-old woman with a background of temporal lobe epilepsy, for which she sees a neurologist on a yearly basis, is referred to a community psychiatrist from primary care. She presents with depressive symptoms and subjective memory impairment. A diagnosis of a moderate depressive episode is made. In addition to the standard biopsychosocial approach to affective disorders, the psychiatric profile of her current anti-epileptic medication (lamotrigine) and the possible effects of antidepressant medication on her seizure threshold are discussed. Her symptoms partially respond to the introduction of a selective serotonin reuptake inhibitor (sertraline) and supportive psychotherapy. Her psychiatrist has the following questions:

- Are any specialist services available for patients with psychiatric disorders associated with neurological disease?

- What information should a referral to behavioural neurology/neuropsychiatry contain?

\section{What is behavioural neurology/ neuropsychiatry?}

The Cartesian mind-body duality is enshrined in medicine by the distinction between organic and functional disorders. Classically, neurology has been concerned with the investigation, diagnosis and management of brain disorders with demonstrable physical signs and cerebral pathology. Psychiatry, on the other hand, has focused primarily on the diagnosis and management of disturbed behaviour, impaired cognition and abnormal affect. Psychiatric disorders were thought to be 'functional' or psychological in origin, i.e. not associated with demonstrable neuropathology. The rise of the depth psychologies introduced by Freud and Jung led to the view that psychological and social processes are the key drivers of mental disturbance. The dominance of the psychosocial approach is still evident in the configuration of mental health services (Bullmore 2009).

However, over the history of British medicine the separation of neurology and psychiatry has not always been so stark. John Hughlings Jackson (1835-1911), a key figure in the development of British neurology and psychiatry (Janković1997), described the psychomotor phenomena associated with temporal lobe epilepsies and proposed the 'dissolution' theory, which would later inform Freud's structural topography of the psyche and the positive-negative dichotomy in schizophrenia. On the European continent, the German neurologist and psychiatrist Wilhelm Griesinger (1817-1868) famously stated that 'mental diseases are brain diseases' (Griesinger 1845). The introduction of antipsychotics (neuroleptics) in the 1950s lent weight to the suggestion that mental diseases are not dissimilar to brain diseases. Then a seminal paper in the mid-1970s demonstrating anatomical differences between the brains of patients with schizophrenia and unaffected controls definitively showed that the psychiatric disturbances of schizophrenia are associated with brain abnormalities (Johnstone 1976).

\section{ARTICLE}

Ketan Dipak Jethwa is currently a CT1 in medicine with Nottingham University Hospitals NHS Trust. Verghese Joseph is a consultant general adult psychiatrist with Lincolnshire Partnership NHS Foundation Trust. Vivek Khosla is a consultant forensic psychiatrist with Oxford Health NHS Foundation Trust. Andrea E. Cavanna is Honorary Reader in Neuropsychiatry and a consultant in behavioural neurology at the Department of Neuropsychiatry, Birmingham and Solihull Mental Health NHS Foundation Trust. He is also Deputy Director for the MSc in Clinical Neuropsychiatry. Correspondence Dr K.D. Jethwa, Nottingham University Hospitals NHS Trust, Nottingham City Hospital, Hucknall, Nottingham NG5 1PB, UK. Email: ketan.jethwa@nuh. nhs.uk 
Over the intervening 40 years significant progress has been made, with an increasing understanding of the genetic and biochemical basis of mental illness. This has led to the development of a specific approach within psychiatry called 'biological psychiatry' (David 2012). The subspecialties of behavioural neurology and neuropsychiatry have emerged (Arzy 2014). Both seek to bridge the ideological gap between neurology and psychiatry and are concerned with understanding disorders of behaviour, affect or cognition in terms of cerebral dysfunction.

The 'cerebral dysfunction' described here is not necessarily structural or localised in classic terms. Psychiatric phenomena have been shown to be associated with neurochemical disturbances and impaired connectivity between widely dispersed brain networks. For example, dysfunction in the salience network, which enables switching between different brain states, has been implicated in the formation of psychotic symptoms (Palaniyappan 2012).

Behavioural neurologists and neuropsychiatrists have special expertise in the diagnosis and management of psychiatric disorders occurring in the context of neurological disease. Teasing apart the aetiological mechanisms of mental illness in patients with neurological comorbidity can be challenging, as both biological factors related to the underlying neurological disorder and psychosocial stressors interact to inform the expression of psychiatric comorbidity.

\section{Who are behavioural neurologists and neuropsychiatrists?}

Behavioural neurologists are physicians who complete core medical training, higher specialist training in neurology and then develop a special interest in the behavioural manifestations of brain pathology. There is no specific training pathway, but interested trainees are encouraged to gain additional clinical experience in established clinics and to engage in subject-specific audits or research. Behavioural neurologists have an important role in considering the relative contributions of organic and psychiatric morbidity and how they interact.

Neuropsychiatrists complete core psychiatric training, higher specialist training in general psychiatry and then develop a special interest in the brain mechanisms underlying psychiatric symptoms. Additional experience in specialist clinics and postgraduate courses such as the MSc in Clinical Neuropsychiatry hosted by the University of Birmingham can complement clinical experience. Neuropsychiatrists, through their psychiatric training, have additional experience of various psychotherapeutic approaches, including cognitive-behavioural therapy and psychodynamic psychotherapy. They also have clinical expertise in the use of the mental health legislation.

In June 2014 the Royal College of Psychiatrists set up the Faculty of Neuropsychiatry. The Faculty expands previous work by the Section of Neuropsychiatry and brings together a number of special interest groups (including the brain injury, epilepsy, sleep, complex neurodisability and movement disorder working groups). It aims to promote neuropsychiatry, develop a curriculum and training pathway, foster research and influence service development.

Behavioural neurologists and neuropsychiatrists work in a number of settings, including National Health Service (NHS) mental health partnership trusts, and they offer an important tertiary-level service. Both specialties are mainly out-patient-based, although in-patient facilities, with multidisciplinary teams, are available in specialist centres in Northampton, Birmingham, North Benfleet (Essex) and Mansfield (Nottinghamshire). These are run by St Andrew's Healthcare, a charity that provides NHS services (www.standrewshealthcare.co.uk). Behavioural neurologists and neuropsychiatrists may also be involved in the medical assessment of patients within the criminal justice system.

\section{What do behavioural neurologists and neuropsychiatrists treat?}

Broadly speaking, behavioural neurologists and neuropsychiatrists are involved in the management of behavioural problems complicating neurological disease that present diagnostic and therapeutic challenges. They are also involved in the multidisciplinary management of patients with 'complex neurodisability'. These are patients who present with multiple neurodevelopmental disorders characterised by motor and behavioural problems. They are usually seen in intellectual disability or child and adolescent mental health services (CAMHS). Behavioural neurologists and neuropsychiatrists are also involved in the assessment and care of patients with functional neurological disorders, including psychogenic seizures and psychogenic movement disorders.

Table 1 summarises the psychiatric comorbidity associated with common neurological conditions. Neuropsychiatric disorders are also associated with more pervasive changes in personality. For example, Geschwind syndrome is a personality syndrome characterised by circumstantiality, altered sexuality and intensified mental life. It is 
classically described in temporal lobe epilepsy, but may also occur in other epilepsy syndromes (Devinsky 1999).

There is currently no comprehensive epidemiological population-based study of the incidence or prevalence of neuropsychiatric comorbidity across all neurological disorders. However, a number of cross-sectional studies across Europe have estimated the prevalence of psychiatric disorders in neurology out-patients at approximately 50\%, with anxiety and depression being the most commonly reported conditions (Carson 2000; Fink 2003). The identification and treatment of psychiatric comorbidity is important to decrease impairment in health-related quality of life, prevent caregiver distress and reduce health and social care costs (Aarsland 1999).

\section{Issues arising from the general adult psychiatry and behavioural neurology/ neuropsychiatry interface}

\section{Identification of neuropsychiatric comorbidity}

The specialisation of psychiatric services arguably means that the era of the truly 'general' psychiatrist is over. Patients with neurological disorders who develop psychiatric problems are often initially referred to general adult psychiatrists by their general practitioner. General adult psychiatrists may instigate initial pharmacotherapy or psychotherapy. Familiarity with the neuropsychopharmacological profiles of medications that cross traditional boundaries between neurology and psychiatry, such as anticonvulsants, is of value (Cavanna 2010; Piedad 2012). The criteria for referral to tertiary services need to be clarified, including recommended psychometric scales to complement clinical assessment.

Disease-specific rating scales are available for use in neuropsychiatric populations and are useful adjuncts to a thorough clinical assessment. In the clinical vignette at the beginning of this article the Neurological Disorders Depression Inventory for Epilepsy (NDDI-E) rating scale could have been used as a valuable screening tool for depression (de Oliveira 2014). Another useful psychometric instrument is the Gilles de la Tourette Syndrome Quality of Life (GTS-QOL) scale, which allows the prioritisation of areas for intervention in adults with tic disorders (Cavanna 2008).

Psychometric instruments that encompass the whole spectrum of behavioural comorbidity in neurology patients are also available, but are often time-consuming and may require specific training before administration. Screening tools encompassing a broad range of neuropsychiatric
TABLE 1 Psychiatric disorders associated with common neurological conditions

\begin{tabular}{|c|c|}
\hline Neurological disease & Commonly comorbid psychiatric disorder \\
\hline Traumatic brain injury & $\begin{array}{l}\text { Anxiety and depression and, less commonly, } \\
\text { mania (Jorge 2014) }\end{array}$ \\
\hline Epilepsy, especially temporal lobe epilepsies & $\begin{array}{l}\text { Anxiety and depression, schizophrenia-like } \\
\text { psychosis (Gaitatzis 2004) and pseudo-seizures }\end{array}$ \\
\hline Sleep disorders, including narcolepsy & Depression \\
\hline $\begin{array}{l}\text { Neurodevelopmental movement disorders, } \\
\text { including tic disorders }\end{array}$ & $\begin{array}{l}\text { Anxiety and depression, obsessive-compulsive } \\
\text { disorder (OCD) and attention-deficit hyperactivity } \\
\text { disorder (Rizzo 2014) }\end{array}$ \\
\hline $\begin{array}{l}\text { Neurodegenerative movement disorders, } \\
\text { including Huntington's disease (chorea) and } \\
\text { Parkinson's disease }\end{array}$ & $\begin{array}{l}\text { Huntington's disease: depression, OCD, } \\
\text { psychosis (De Marchi 2000) } \\
\text { Parkinson's disease: depression, psychosis, } \\
\text { cognitive impairment and impulse control } \\
\text { disorders (Factor 2014; Sagna 2014; Zhang 2014) }\end{array}$ \\
\hline
\end{tabular}

symptoms could be of use in general psychiatry or neurology clinics, where the initial focus is on the identification of comorbidity. In this context the Neuropsychiatric Inventory (NPI) is a useful screening tool to determine the extent of comorbid psychopathology. The NPI assesses ten different domains of behavioural disturbance, including delusions, hallucinations, agitation, depression, anxiety, elation, apathy, disinhibition, irritability, abnormal motor behaviour, sleep disturbance and eating/appetite problems (Cummings 1994). This instrument reduces administration time by having screening questions for each symptom domain: positive responses are explored further, with assessment of the frequency and severity of relevant symptoms. The questionnaire is completed by a caregiver familiar with the patient, so changes in behaviour from baseline can be determined and an indirect assessment of caregiver burden can be obtained.

\section{Care pathways and accessibility of neuropsychiatric services}

Protocols regarding the appropriate identification and referral of patients with neurological disease and psychiatric comorbidity can be devised and agreed by medical advisory committees in NHS mental health trusts. These protocols should include a thorough clinical assessment with the use of supporting psychometric rating scales. Protocols and care pathways should be devised collaboratively, with an agreement to audit and review processes annually.

There is growing consensus in the tertiary neuropsychiatry community that out-patient neuropsychiatry services might expect a referral rate of 20-30 patients with comorbid neurological and psychiatric disorders per 100000 population per year (Agrawal 2008). 


\section{$\mathrm{MCO}$ answers}

$1 d \quad 2 b \quad 3 e \quad 4 b \quad 5 b$

\section{Accessibility of neuropsychiatry services}

There is currently remarkable variability in the availability of neuropsychiatrists across the UK. Ideally, behavioural neurologists and neuropsychiatrists would be readily available for advice on specific cases, by written communication, telephone or invitation to general adult clinical reviews. However, given the limited availability of specialists and their concentration in centres of excellence (such as London, Birmingham and Edinburgh), it is unlikely that a behavioural neurologist or neuropsychiatrist will always be available at short notice. To tackle these operational hurdles, a 'hub-and-spoke' model for neuropsychiatry services has been proposed (Agrawal 2008). This would include a regional neurosciences centre with outreach clinics and support offered to general psychiatrists with a special interest in neuropsychiatry. This model has been successfully implemented with such a clinic (Leonard 2002). It has also been suggested that an out-patient neuropsychiatry service, in hub, should include at least two consultants, one junior doctor or associate specialist, one neuropsychologist, one counsellor, one nurse specialist and one administrator (Agrawal 2008).

Neuropsychiatric services are rapidly developing, but progress has been stifled by variation in funding, given that NHS neurology and psychiatry services tend to be delivered by separate trusts or health boards. At present there is considerable variation in accessibility and referral pathways across the UK, although most healthcare providers would favour progression to hub-and-spoke service configurations, with referrals from primary and secondary care to tertiary centres and outreach services in the community. For brief reviews of the historical background and potential developments in behavioural neurology and neuropsychiatry in the UK readers might refer to Agrawal et al (2008), Leonard et al (2002) and Arambepola et al (2012).

\section{Conclusions}

This is an introductory article exploring the working links between general psychiatry and the specialty of behavioural neurology/ neuropsychiatry. It aims to help readers identify disorders commonly encountered in neuropsychiatry and behavioural neurology. Closer collaboration between psychiatrists and behavioural neurologists/neuropsychiatrists is required for the timely diagnosis and effective treatment of patients with comorbid neurological and psychiatric disorders. It is therefore important that links between neurology and psychiatry are incorporated within training pathways to ensure that general adult psychiatrists can acquire the necessary skills to identify and appropriately refer patients to tertiary services.

\section{References}

Aarsland D, Larsen JP, Karlsen K, et al (1999) Mental symptoms in Parkinson's disease are important contributors to caregiver distress. International Journal of Geriatric Psychiatry, 14: 866-74.

Agrawal N, Fleminger S, Ring H, et al (2008) Neuropsychiatry in the UK: planning the service provision for the 21st century. Psychiatrist, 32 : 303-6.

Arambepola NMA, Rickards H, Cavanna AE (2012) The evolving discipline and services of neuropsychiatry in the United Kingdom. Acta Neuropsychiatrica, 24: 191-8.

Arzy S, Danziger S (2014) The science of neuropsychiatry: past, present and future. Journal of Neuropsychiatry and Clinical Neurosciences, 26: 392-5.

Bullmore E, Fletcher P, Jones PB (2009) Why psychiatry can't afford to be neurophobic. British Journal of Psychiatry, 194: 293-5.

Carson AJ, Ringbauer B, Stone J, et al (2000) Do medically unexplained symptoms matter? A prospective cohort study of 300 new referrals to neurology outpatient clinics. Journal of Neurology, Neurosurgery, and Psychiatry, 68: 207-10

Cavanna AE, Schrag A, Morley D, et al (2008) The Gilles de la Tourette syndrome-quality of life scale (GTS-0OL): development and validation. Neurology, 71: 1410-6.

Cavanna AE, Ali F, Rickards HE, et al (2010) Behavioural and cognitive effects of anti-epileptic drugs. Discovery Medicine, 9: 138-44.

Cummings JL, Mega M, Gray K, et al (1994) The Neuropsychiatric Inventory: comprehensive assessment of psychopathology in dementia. Neurology, 44: 2308-14.

David A, Fleminger S, Kopelman M, et al (2012) Lishman's Organic Psychiatry: A Textbook of Neuropsychiatry (4th edn). Wiley-Blackwell.

De Marchi N, Mennella R (2000) Huntington's disease and its association with psychopathology. Harvard Review of Psychiatry, 7: 278-89.

de Oliveira GN, Lessa JM, Gonçalves AP, et al (2014) Screening for depression in people with epilepsy: comparative study among neurological disorders depression inventory for epilepsy (NDDI-E), hospital anxiety and depression scale depression subscale (HADS-D), and Beck depression inventory (BDI). Epilepsy \& Behavior, 34: 50-4.

Devinsky 0, Najjar S (1999) Evidence against the existence of a temporal lobe epilepsy personality syndrome. Neurology, 53 (suppl 2): S13-25.

Factor SA, Scullin MK, Sollinger AB, et al (2014) Cognitive correlates of hallucinations and delusions in Parkinson's disease. Journal of the Neurological Sciences, 347: 316-21.

Fink P, Hansen MS, Søndergaard L, et al (2003) Mental illness in new neurological patients. Journal of Neurology, Neurosurgery \& Psychiatry, 74: 817-9.

Gaitatzis A, Trimble MR, Sander JW (2004) The psychiatric comorbidity of epilepsy. Acta Neurologica Scandinavica, 110: 207-20.

Griesinger W (1845) Die Pathologie und Therapie der psychischen Krankheiten [Mental Pathology and Therapeutics]. Stuttgart.

Janković SM, Sokić DV, Lević Z, et al (1997) [Dr John Hughlings Jackson]. Srpski arhiv za celokupno lekarstvo [Serbian Archives of Medicine], 125: 381-6.

Johnstone EC, Crow TJ, Frith CD, et al (1976) Cerebral ventricular size and cognitive impairment in chronic schizophrenia. Lancet, 2: 924-6.

Jorge RE, Arciniegas DB (2014) Mood disorders after TBI. Psychiatric Clinics of North America, 37: 13-29.

Leonard J, Majid S, Sivakumar K (2002) Service innovations: a neuropsychiatry outreach clinic. Psychiatric Bulletin, 26: 99-101.

Palaniyappan L, White TP, Liddle PF (2012) The concept of salience network dysfunction in schizophrenia: from neuroimaging observations to therapeutic opportunities. Current Topics in Medicinal Chemistry, 12 : 2324-38. 
Piedad J, Rickards H, Besag FM, et al (2012) Beneficial and adverse psychotropic effects of antiepileptic drugs in patients with epilepsy: a summary of prevalence, underlying mechanisms and data limitations. CNS Drugs, 26: 319-35.

Rizzo R, Gulisano M, Pellico A, et al (2014) Tourette syndrome and comorbid conditions: a spectrum of different severities and complexities. Journal of Child Neurology, 29: 1383-9.
Sagna A, Gallo JJ, Pontone GM (2014) Systematic review of factors associated with depression and anxiety disorders among older adults with Parkinson's disease. Parkinsonism and Related Disorders, 20: 708-15

Zhang G, Zhang Z, Liu L, et al (2014) Impulsive and compulsive behaviors in Parkinson's disease. Frontiers in Aging Neuroscience, 6: 318.

\section{MCQs}

Select the single best option for each question stem

1 Which of the following statements is false?

a Depression is common in patients with epilepsy

b Huntington's disease is associated with choreiform movements

c Traumatic brain injury is often associated with depression and anxiety

d Alzheimer's disease never requires input from a behavioural neurologist/neuropsychiatrist

e Tic disorders are associated with behavioural problems in the vast majority of cases.

\section{Which of the following statements is} false?

a Behavioural neurologists/neuropsychiatrists can work in both out-patient and in-patient settings

b Psychiatric phenomena are not associated with disturbed neural networks

c Behavioural neurologists/neuropsychiatrists are currently based in specialist centres throughout the UK $d$ The cost per patient per year is different for general adult and behavioural neurology/ neuropsychiatry services

e Behavioural neurologists/neuropsychiatrists are often involved in forensic consultations.

3 Which of the following statements is true?

a Behavioural neurologists initially train as psychiatrists

b Neuropsychiatrists initially train as physicians

c Behavioural neurologists/neuropsychiatrists do not have access to multidisciplinary teams

d Behavioural neurology/neuropsychiatry is a secondary service

e There are psychometric rating scales for assessing depressive symptoms in epilepsy.

4 An appropriate primary reason for referral to behavioural neurology/neuropsychiatry is that:

a the patient poses a suicide risk

b the patient has depression and develops episodes of cataplexy and sleep paralysis c the patient has an 10 below 70 $\mathrm{d}$ the patient has a history of epilepsy and substance misuse

e the patient has a history of childhood epilepsy which has completely resolved.

5 Which of the following is not likely to help resolve disagreements at the interface between general adult psychiatry and behavioural neurology/neuropsychiatry?

a A protocol for the management of patients across services

b No explicit referral criteria

c Regular liaison among colleagues

$\mathrm{d}$ An audit of referrals to behavioural neurology/ neuropsychiatry

e Serious case reviews. 